\title{
The Biological-promoting Function of Dietary Nano- selenium, Seaweed Gracilaria Lemaneiformis and Nano-selenium-enriched Gracilaria Lemaneiformis on Growth Performance, Antioxidant and Immunity Systems and Meat Quality of Grouper (Epinephelus Coioides)
}

Changpeng Ye ( $\nabla$ cpye@163.ocm )

Jinan University https://orcid.org/0000-0002-3060-6252

Jiaojiao Yu Jinan University

Yuying Huang Jinan University

Chaowen Zhang Jinan University

Min Wu Jinan University

Zibin Wang Jinan University

Yi Zou Jinan University

Jiangang Zhao

Jinan University

Delin Xu

Jinan University

Miao Cui

Jinan University

Research

Keywords: Epinephelus coioides, Nano-Se, Gracilaria lemaneiformis, Growth, Immune

Posted Date: April 30th, 2021 
DOl: https://doi.org/10.21203/rs.3.rs-472856/v1

License: (c) (1) This work is licensed under a Creative Commons Attribution 4.0 International License. Read Full License 


\section{Abstract}

Background: Nano-Se-enriched Gracilaria lemaneiformis (G. lemaneiformis) as feed ingredient on aquatic animals remained largely unknown. Feeding experiment was conducted for a total of 45 days to research the biological impacts of nano-selenium (Nano-Se) (group N), G. lemaneiformis (group G) and Nano-Seenriched G. lemaneiformis (group NG) on growth, selenium concentration, oxidation, immunity and nutrition of Epinephelus coioides.

Results: At each tested time point, the weight gain rate, specific growth rate and feed conversion rate of group N, group $\mathrm{G}$ and group NG were significantly increased in contrast to those of the control group (group C). A remarkable increase of the hepatosomatic index (HSI) and viscerosomatic index (VSI) was observed in group $\mathrm{N}$ and group NG. The activities of superoxide dismutase (SOD), catalase (CAT) and glutathione peroxidase (GPx) of fish in group N, group $\mathrm{G}$ and group NG were significantly enhanced relative to those in group $\mathrm{C}$. The immune system was also activated by showing an elevated expression level of various immunological genes including Immunoglobulin $M$, tumor necrosis factor-a and Interleukin -8. The muscle Se content in group N and group NG was 31- and 40.5-fold that of the control group (group C), respectively. The liver Se value of group N and group NG was 1.06 and 2.50 times higher than that of group $C$, respectively. The lipid and moisture values of group $N$ and group NG are increased and reduced, respectively, in comparison to that of group C. The diet supplementation of Nano-Seenriched $G$. lemaneiformis led to significant increase of the protein content, delicious amino acids along with total amino acids content in grouper.

Conclusions: Our results demonstrate a great application potential of Nano-Se-enriched G. lemaneiformis as dietary additive in grouper.

\section{Background}

For fish, Selenium (Se) is an indispensable microelement to maintain normal growth. It is an absolutely necessary part of the active center of glutathione peroxidase, which plays important roles in reducing excess free radicals accumulated in stressed fish [1]. Appropriate supply of Se in diet is critical for the normal development of fish, and it has been demonstrated that inappropriate supply of selenium negatively affected the integrity of immune organs [2]and/or cause poisonous death in fish [3]. Nano selenium (Nano-Se) in unique nanometer size is known with excellent catalytic activity [4], and has been widely used in dietary supplements because of its low toxicity and high bioavailability [5]. Studies have shown that the addition of Nano-Se in diet led to energetic effects on the growth and antioxidant defense system in carp [6], immunity in rainbow trout [7], and meat quality in blunt snout bream [8].

The red seaweed Gracilaria lemaneiformis (G. lemaneiformis) are cultivated on a large scale in southern China [9], which contains polysaccharides, minerals, vitamins, proteins and other bioactive substances [10]. G. lemaneiformis is known with excellent Se enrichment ability [11]. Se can promote the activity of functional substances such as polysaccharides in $G$. lemaneiformis by modifying its structure $[12,13]$. It 
is feasible and effective to use proper $G$. lemaneiformis as feed ingredient for juvenile Pacific white shrimp [14]. The application potential of Nano-Se-enriched G. lemaneiformis as feed additives have been proposed in terms of the economic point and nutritional value [11], however, the specific influences of Nano-Se-enriched $G$. lemaneiformis as feed ingredient on aquatic animals remained largely unknown.

Grouper (Epinephelus coioides) is one of the principal cultured economic fish in China, known with desired meat quality and high nutritional value [15]. Intensive fish farming has been shown to cause stress responses widely and subsequently led to severe disease and death, with the resulting decline in fish quality [16]. Therefore, it is in urgent need to develop highly effective feed additives so as to achieve better growth in grouper. In the present study, the dietary Nano-Se, G. lemaneiformis, and Nano-Seenriched $G$. lemaneiformis were applied as feed ingredient, and their biological effects including growth performance, antioxidant ability, immune response and nutritional quality of grouper (Epinephelus coioides) were explored. The results indicate a great application potential of Nano-Se and seaweeds in aquaculture and provide a new low-cost feed additive candidate for fish.

\section{Methods}

\section{Feed pretreatment}

G. lemaneiformis was collected from the same batch of artificial cultivation in Xiamen, Fujian Province, China. Nano-Se was purchased from Guangdong Jichuang Selenium Nano Research Institute Co.Ltd. G. lemaneiformis were cleaned with natural seawater to remove the epiphytes, and acclimated in the aquarium filled with natural seawater at $25^{\circ} \mathrm{C}$ for three days. $2000 \mathrm{~g} \mathrm{G}$. lemaneiformis were randomly divided into two equal portions and then placed in two $100 \mathrm{~L}$ fish tanks at $25^{\circ} \mathrm{C}$. Nano-Se was introduced into one tank at a final concentration of of $1500 \mathrm{mg} / \mathrm{L}$ for Se enrichment, while the other tank without any supplementation was supplied as the control. After three days of culture, G. lemaneiformis were cleaned with natural seawater, and then subjected to drying, followed by grinding. The resultant $G$. lemaneiformis powder was stored at room temperature until diet preparation.

The diet was purchased from Guangdong Yuequn Marine Biological Research and Development Co. Ltd. 0.7mg Nano-Se/kg-diet (group N), $1 \%$ dried G. lemaneiformis powder (group G) and $1 \%$ Nano-Se-enriched dried $G$. lemaneiformis powder (group NG) were separately supplemented into the basal diet, and the group without any addition was supplied as the control (Table 1) [14, 17]. The prepared diet was stored at $4^{\circ} \mathrm{C}$ until further applications.

\section{Fish maintenance and treatment}

Grouper were collected from Guangdong Marine Fishery Experimental Center and allowed to acclimate for one week at $25.2 \pm 0.1^{\circ} \mathrm{C}$ in natural seawater. Groupers were fed with basic feed twice daily under a photoperiod of 12 hours of light: 12 hours of dark. The seawater for farmed grouper was renewed every other day. After the preliminary acclimation, 240 fish ( $80.89 \pm 0.23 \mathrm{~g}$ in average weight) were randomly picked and evenly transferred to 12 filter circulation aquariums $(810 \times 365 \times 700 \mathrm{~mm}, 150 \mathrm{~L}, 20 \mathrm{fish}$ per 
aquarium). Fish were fed one of the four diets (Table 1) in triplicate groups at 8:30 and 18:30 every day, with a daily feed equivalent to $3 \%$ of their body weight. The body weight of grouper was measured once a week, and then the daily feed amount was updated. The remains of bait and feces were separately collected 1-2 hours post feeding and weighed. Throughout the experimental period of 45 days, the natural seawater was maintained at $25.2 \pm 0.1^{\circ} \mathrm{C}, \mathrm{pH} 7.1 \pm 0.05$, with dissolved oxygen of $5.8 \pm 0.5 \mathrm{mg} / \mathrm{L}$. 12 -hour light: 12-hour dark photoperiod was used for breeding. One half of the seawater in each aquarium was renewed every 3 days to ensure the water quality.

\section{Samples collection}

Five fish from each aquarium were sampled on the $0^{\text {th }}, 15^{\text {th }}, 30^{\text {th }}$ and $45^{\text {th }}$ day during fish rearing, and subjected to the analysis of weight gain rate (WG), specific growth rate (SGR), feed conversion ratio (FCR) and condition factor (CF). The tail vein blood of grouper was collected with a sterile syringe for later determination of serum antioxidant enzyme activities. The liver and total viscera of the groupers were separated with a sterile scalpel, and then weighed to calculate the hepatosomatic index (HSI) and viscerosomatic index (VSI). The gills, spleen, kidney and intestines of grouper were stripped and frozen immediately in liquid nitrogen for the analysis of immune-related genes. At the $45^{\text {th }}$ day, the muscle and liver was stripped for analyzing Se concentration and nutrient composition.

\section{Analytical methods}

The Se level in the diets, muscle and liver was measured by inductively coupled plasma mass spectrometry (ICP-OES, thermo iCAP 7400 series, USA). The calculation formula of growth performance is as follows [9]:

WG $(\%)=[$ final body weight $(\mathrm{g})$ - initial body weight $(\mathrm{g})] /$ initial body weight $(\mathrm{g}) \times 100$;

SGR $\left(\%\right.$ day $\left.^{-1}\right)=[($ In final body weight $(\mathrm{g})-$ In initial body weight $(\mathrm{g})] /$ days $\times 100$;

$\mathrm{CF}(\%)=$ final body weight $(\mathrm{g}) /$ total length $^{3}(\mathrm{~cm}) \times 100$;

FCR= [final body weight $(\mathrm{g})$ - initial body weight $(\mathrm{g})] /$ dry feed consumed $(\mathrm{g})$

HSI (\%) = liver weight $(\mathrm{g}) /$ whole body weight $(\mathrm{g}) \times 100$;

VSI $(\%)=$ visceral weight $(\mathrm{g}) /$ whole body weight $(\mathrm{g}) \times 100$.

The superoxide dismutase (SOD), catalase (CAT) and glutathione peroxidase (GPx) were detected by Total SOD Assay Kit with NBT, CAT Assay Kit, Cellular GPx Assay Kit with NADPH, respectively, as described in previous studies [18]. All assay kits were purchased from Beyotime Biotechnology. Total RNA of gills, spleen, kidney and intestines were collected by Trizol reagent (Beyotime, China) according to the manufacturer's instructions. The mRNA levels of immunoglobulin M ( $\mathrm{IgM})$, tumour necrosis factor-a (TNF-a), interleukin-8 (IL-8) were analyzed using a BeyoRT ${ }^{T M}$ II First Strand cDNA Synthesis Kit and a Real 
Time PCR System. Primers specific for these genes were listed in Table 2. The moisture (GB 5009.3-2016, China), ash (GB 5009.4-2016, China), lipid (GB 5009.5-2016, China), protein (GB 5009.6-2016, China) and amino acid of muscle (GB 5009.9-2016, China) were determined by Guangzhou Kingmed Testing Science \& Technology Co., Ltd, (Guangzhou, China).

\section{Statistical analysis}

All the data are displayed in the form of mean values \pm SD. Excel 2010 and SPSS 16.0 were used to process the experimental data. One-way ANOVA and the least Significant Difference (LSD) multiple comparison tests were used to reveal the significance between different treatment groups at the same sampling time, and the significance of different sampling time in the same treatment group. $p<0.05$ represents a significant difference.

\section{Results}

\section{Se concentration}

In Nano-Se supplemented group, Se content in muscle and liver was 32 and 2.06 times higher than that in the control group, respectively $(p<0.05)$ (Table 3$)$. While in Nano-Se-enriched seaweed supplemented group, a better Se accumulation was detected in the tissues of muscle and liver, which was 41.5 and 3.49 times higher than that in the control group, respectively $(p<0.05)$. No striking difference was discovered in terms of the Se content upon G. lemaneiformis supplementation.

\section{The growth performance}

WG, SGR, and FCR of the grouper in various groups generally showed an increasing manner with the ongoing of cultivation. At each specific time point, the values of WG, SGR, and FCR in the Se and/or seaweed supplemented groups were always larger than that in the group without supplementation $(p<$ 0.05) (Fig.1). While, the highest value of WG, SGR, and FCR were obtained in group NG on the $45^{\text {th }}$ day ( $p$ $<0.05)$, followed by that in group $\mathrm{N}$ and group $\mathrm{G}$. Interestingly, CF was not affected by either treatment group.

\section{Hepato-pancreatic index}

At each specific time point, VSI and HSI in group NG and N were higher than that in group $\mathrm{C}(p<0.05)$ (Fig. 2). And the utmost value of VSI and HSI were observed in group NG on the $45^{\text {th }}$ day $(p<0.05)$, which were 1.19 and 1.48 times higher than those on the $0^{\text {th }}$ day, respectively. In contrast, no notable distinction was observed between groups $\mathrm{G}$ and group $\mathrm{C}$.

\section{Antioxidant enzyme activity}

The enzyme activities of SOD, CAT, and GPx increased with the ongoing of culturing time. The values of SOD and CAT reached a peak on the $30^{\text {th }}$ day, and then declined at the following time point. In each 
specific group, the activities of the enzymes in the Se and/or seaweed supplemented groups were always higher than that in the group without extra supply. (Fig.3). A maximum value of SOD and CAT appeared in group NG on the $30^{\text {th }}$ day $(p<0.05)$, which was 4.70 and 1.56 times higher than those on the $0^{\text {th }}$ day. In contrast, the GPx activity in group $N$ and group NG reached a highest value on the $45^{\text {th }}$ day, which was 2.15 and 2.27 times higher than that on the $0^{\text {th }}$ day, respectively $(p<0.05)$.

\section{Immune response}

Upon the feeding of the diet suppled with Nano-Se, seaweed and Nano-Se-enriched seaweed, the relative expression pattern of IgM, IL-8 and TNF-a mRNA in various tissues of gills, spleen, kidney and intestines showed an increase first, and reached a peak on the $30^{\text {th }}$ day, followed by a decline (Fig.4). At each of the specific sampling point, the immune genes upon any of the three treatments were significantly upregulated in the tested tissues in comparison to that in the control group $(p<0.05)$. Meat quality

\section{Composition of muscles}

The lipid and moisture content were significantly increased and reduced respectively in the muscles of the two supplemental Se diet groups compared with that in the groups without extra Se $(p<0.05)$, however, the ash content were not influenced by any dietary supplementation (Table 4 ). The maximum protein content appeared in group NG, which was 1.19 times higher than that in the control group $(p<$ 0.05).

\section{Amino acid composition}

A total of 16 common amino acids were detected in the muscle of four groups (Table 5), including 7 essential amino acids (Thr, Val, Met, lle, Leu, Phe and Lys), 2 semi-essential amino acids (His and Arg) and 7 non-essential amino acids (Asp, Glu, Gly, Ala, Tyr, Pro and Ser). Tryptophan was destroyed during the hydrolysis process and was therefore excluded from measuring. The total delicious amino acids $\left(\mathrm{W}_{\text {DAA }}\right)$, total essential amino acids ( $\left.\mathrm{W}_{\text {EAA }}\right)$, total non-essential amino acids ( $\mathrm{W}_{\text {ENAA }}$ ) and total amino acids ( $\mathrm{W}_{\text {TAA }}$ ) of the three treatment groups of fish muscles were significantly enhanced by dietary supplementation $(p<0.05)$. The $\mathrm{W}_{\text {EAA }} / \mathrm{W}_{\mathrm{TAA}}$ of the four groups of fish muscles were $41 \%, 41 \%, 40 \%$ and $42 \%$, respectively. The $\mathrm{W}_{\text {EAA }} / \mathrm{W}_{\mathrm{NEAA}}$ of the four groups of fish muscles were $71 \%, 70 \%, 68 \%$ and $72 \%$, respectively.

\section{Discussion}

The Se concentration in grouper fed the dietary with Nano-Se-enriched G. lemaneiformis was much higher than that in grouper fed the dietary with Nano-Se. The results clearly demonstrated that Nano-Se-enriched G. lemaneiformis is more advantageous in promoting tissue enrichment of Se. This phenomenon might be interpreted by the differential metabolic pathways of Nano-Se and Nano-Se-enriched G. lemaneiformis in grouper [19]. It is known that Se in the form of either Nano-Se or Nano-Se-enriched G. lemaneiformis would be metabolized into active Se, which could then be subjected to the synthesis of selenase. 
However, in terms of Nano-Se-enriched G. lemaneiformis, preservation might occurred in other proteins as well in the form of selenomethionine [20]. This indicated that Nano-Se-enriched G. lemaneiformis could be more effective than Nano-Se in deposition of Se in grouper.

In this paper, after 45 days of dosing, the WG, SGR and FCR were clearly higher in dietary supplement group than those in the control group. This is not consistent with the previously demonstrated results in rainbow trout since no significant difference was observed in growth performance upon either inorganic Se or organic Se supplementation[21]. This could be due to the discrepancies of feed conversion rates, bioavailability of Se in different forms, the dose of Se and the species of fish between the two studies. It has been indeed demonstrated that the bioavailability of inorganic Se or organic Se was not as high as Nano-Se [22]. Our results showed that Nano-Se-enriched G. lemaneiformis supplementation gave a better promotion effects to growth performance as well as Se accumulation ability in grouper. This could be resulted from the presence of both inorganic and organic forms of Se in Nano-Se-enriched $G$. lemaneiformis, or from the cooperation between Nano-Se and the G. lemaneiformis harbored multifunctional components (such as polysaccharides) [12]. It was previously reported that the fiber in $G$. lemaneiformis can inhibit the growth of carnivorous fish by reducing the pepsin activity or lipid digestibility $[9,23]$. While conversely in this paper, the dietary $G$. lemaneiformis was shown to promote growth, indicating that $1 \%$ dietary supplement level is suitable for grouper.

$\mathrm{HSI}$ and VSI of fish were influenced by $0.7 \mathrm{mg} / \mathrm{kg}$ Nano-Se and $1 \%$ Nano-Se-enriched G. lemaneiformis, but not by $1 \%$ G. lemaneiformi. These indicate that dietary Nano-Se and Nano-Se-enriched $G$. lemaneiformis was better than dietary $G$. lemaneiformis in promoting immune function, since it is generally acknowledged that the immunity of the organism is related to the development of immune organs [13] . On the other hand, elevation of HSI reflected an increase in energy storage in grouper fed the dietary with Nano-Se or Nano-Se-enriched G. lemaneiformis [24], which is consistent with the result of weight gain in the same groups.

Upon external or internal pressure, fish is known to produce large amounts of free radicals, and the excessive amount of which can cause damage to fish health [25]. The fluctuation of antioxidant enzyme activity reflects oxidative stress in aquatic products [26]. The results in this study demonstrated that the activity of GPx, SOD, and CAT were significantly increased by three supplemental dietary, indicating a promoted blood serum antioxidant activity of grouper. Moreover, SOD and CAT of grouper in three groups fed supplemental dietary stated clearly a curve of increasing first and then slightly declining during the experiment, and the maximum appears on the $30^{\text {th }}$ day. This curve indicated that all three dietary supplementation meet the needs for maximizing SOD and CAT activity on the $30^{\text {th }}$ day, however, due to many complex factors such as the feedback inhibition of antioxidant systems and the life stage of fish bodies, the activity of antioxidant enzymes in grouper decreased slightly on the $45^{\text {th }}$ day. In contrast, the GPx activity kept showing an increasing trend upon the Nano-Se dietary or the Nano-Se-enriched $G$. lemaneiformis dietary. Considering the fact that GPx activity is an index to judge Se content [27], the observed increasing trend might be due to the elevated Se content as illustrated in Table 3. Interestingly, the GPx activity of grouper fed with Nano-Se-enriched G. lemaneiformis was greater than that of fed with 
Nano-Se. This might be associated with free radical scavenging from biological activities (such as $\mathrm{Se}$ polysaccharides and phycoerythrin) in Nano-Se-enriched G. lemaneiformis [28]. This result indicate that Nano-Se-enriched G. lemaneiformis is more capable of enhancing the antioxidant defense systems.

To our knowledge, an appropriate increase in cytokines (such as TNF-a and IL-8) is conducive to macrophages and neutrophils exert immune function, and further fight off stress caused by various reasons [29]. In fish, IgM is secreted by lymphocyte $B$, which mediates humoral immunity [30]. The current results propose that dietary Nano-Se, G. lemaneiformis and Nano-Se-enriched G. lemaneiformis can stimulate the immune response by up regulating the expression of IgM, IL-8 and TNF-a, thus improving the defense ability of fish against bacterial harm in the process of fish culture. This might be associated with the activation of TNF-a signaling pathways such as NF-KB [31], or the stimulation of macrophages and neutrophils as illustrated previously $[30,32]$. It has been proposed that TNF-a affects fish immunity by regulating the expression of other cytokines including IL-8 [32]. And our results provide further evidence for this regulatory link. The transcription level of IgM, IL-8 and TNF-a in grouper was activated to a highest level upon the feeding of Nano-Se-enriched G. lemaneiformis supplemented diet, indicating a mutual cooperation between Se and seaweed in promoting immune system, which is consistent with the previous demonstrated results of immune organ index and antioxidant enzyme activity (Fig. $2 \& 3$ ).

Lipid and protein are widely acknowledged indexes to evaluate the nutritional status of fish [33]. The results in this paper demonstrated that lipid was markedly increased in the muscle of grouper fed containing Nano-Se and Nano-Se-enriched G. lemaneiformis than that in the control. Lipid of grouper could be influenced by the conversion and utilization of diet [34], which was proved by the elevated FCR with Se-contained feed in this study (Fig.1). The protein content of grouper muscle was increased by dietary Nano-Se-enriched G. lemaneiformis. However, it was reported that supplementation with Se has no effect on the protein of fish meat $[6,7]$. The differences might be caused by the discrepancies of the form and dose of Se, or the specific fish species applied, since it has been demonstrated that grouper has high bioavailability to Nano-Se-enriched G. lemaneiformis (Table 3).

Fish is known with high-level protein which provides essential amino acids [8]. According to the data released from Food and Agriculture Organization/World Health Organization (FAO/WHO), the ideal protein should meet the following standards: $\mathrm{W}_{\text {EAA }} / \mathrm{W}_{\text {TAA }}$ is about $40 \%$, and $\mathrm{W}_{\text {EAA }} / \mathrm{W}_{\text {NEAA }}$ is more than $60 \%$. The result of present study indicates that the muscle amino acid composition met the criterion of the ideal model of FAO/WHO in the four groups of groupers. Furthermore, $\mathrm{W}_{\mathrm{DAA}}$ that responsible for the umami taste in grouper was increased significantly in three dietary supplement groups than that in the control, which is expected to guarantee a better taste quality. WAA $_{\text {EA }}$ of grouper were increased by either form of Se supplementation, but not by $G$. lemaneiformis supplemented diets, indicating a decisive role of Se in improving meat quality. In addition, $\mathrm{W}_{\text {EAA }}, \mathrm{W}_{\mathrm{NEAA}}, \mathrm{W}_{\text {DAA }}$ and $\mathrm{W}_{\text {TAA }}$ were obviously greater in grouper fed the dietary with Nano-Se-enriched $G$. lemaneiformis than in grouper fed the dietary with NanoSe, which once again provides a basis for the higher bioavailability of grouper to Nano-Se-enriched $G$. lemaneiformis than Nano-Se. 


\section{Conclusions}

In closing, the growth, antioxidant capacity, immunity and meat quality of grouper were significantly improved by dietary Nano-Se, G. lemaneiformis and Nano-Se-enriched G. lemaneiformis, while Nano-Seenriched $G$. lemaneiformis supplemented group was always with better performance, and therefore has stronger application potential as nutritional additives. It would be meaningful to analyze the active components of Nano-Se-enriched G. lemaneiformis, so as to better understand the mechanism underlying the activating phenotype.

\section{Abbreviations}

Nano-Se: nano-selenium; G. lemaneiformis: Gracilaria lemaneiformis; Se: Selenium; WG: weight gain rate; SGR: specific growth rate; FCR: feed conversion ratio; CF: condition factor; HSI: hepatosomatic index; VSI: viscerosomatic index; SOD: superoxide dismutase; CAT: catalase; GPx: glutathione peroxidase; IgM: immunoglobulin M; TNF-a: tumour necrosis factor-a; IL-8: interleukin-8; LSD: least Significant Difference; Thr: treonine; Val: valine; Met: methionine; Ile: isoleucine; Leu, leucine; Phe: phenylalanine; Lys: lysine; His: hlstidine; Arg: argnine; Asp: aspartic acid; Glu: glutamic acid; Gly: glycine; Ala: alanine; Tyr: tyrosine; Pro: proline; Ser: serine; WDAA: total delicious amino acids; WEAA: total essential amino acids; WENAA: total non-essential amino acids; WTAA: total amino acids; FAO: Food and Agriculture Organization of the United Nations; WHO: World Health Organization.

\section{Declarations}

\section{Acknowledgements}

The authors thank those who participated in this experiment.

\section{Authors' contributions}

Changpeng Ye, Delin Xu and Miao Cui designed the study, Jiangang Zhao provided technical assistance, Yi zou funded the study. Jiaojiao Yu and Yuying Huang, Chaowen Zhang started breeding work. Jiaojiao Yu, Zibin Wang and Min Wu measured the experimental data. Jiaojiao Yu analyzed the results and wrote the paper.

\section{Funding}

Thanks for the State Key Program of National Natural Science of China (42030713), Special Fund for Marine Economic Development in Guangdong Province (2020035), the Science and Technology Planning Project of Guangzhou (201804010494; GZKTP202032), Rural Science \& Technology to the countryside in 2019 to find problems condensed science and technology to help agriculture project (32419078), Guangdong Marine and Fishery Bureau Science and Technology Project (SDYY-2018-08).

\section{Availability of data and materials}


The datasets analysed in the current study are available from the corresponding author on reasonable request.

\section{Ethics approval and consent to participate}

The experimental program and procedure are approved by the Experimental Animal Ethics Committee of Jinan University under permit No.SYXK 2017-0174.

\section{Consent for publication}

Not applicable.

\section{Competing interests}

The authors declared that they have no known competing interests in the work reported in this paper.

\section{Author details}

${ }^{1}$ Institute of Hydrobiology of Jinan University, Guangzhou, Guangdong Province 510632, China.

${ }^{2}$ Department of Biology, School of life Science, Jinan University, Guangzhou, Guangdong Province 510632, China.

\section{References}

1. Antony Jesu Prabhu P, Schrama J W, Kaushik S J. Mineral requirements of fish: a systematic review. Reviews in Aquaculture. 2016;8(2):172-219. https://doi.org/10.1111/raq.12090

2. Zheng $L$, Jiang $W D$, Feng $L$, Wu $P$, Tang $L$, Kuang $S Y$, et al. Selenium deficiency impaired structural integrity of the head kidney, spleen and skin in young grass carp (Ctenopharyngodon idella). Fish Shellfish Immunol. 2018;82:408-420. https://doi.org/10.1016/j.fsi.2018.08.038

3. Kumar N, Krishnani K K, Singh N P. Comparative study of selenium and selenium nanoparticles with reference to acute toxicity, biochemical attributes, and histopathological response in fish. Environ Sci Pollut Res Int. 2018;25(9):8914-8927. https://doi.org/10.1007/s11356-017-1165-x

4. Alphandéry E. A discussion on existing nanomedicine regulation: Progress and pitfalls. Applied Materials Today. 2019;17:193-205. https://doi.org/10.1016/j.apmt.2019.07.005

5. Dawood M A O, Koshio S, Zaineldin A I, Van Doan H, Moustafa E M, Abdel-Daim M M, et al. Dietary supplementation of selenium nanoparticles modulated systemic and mucosal immune status and stress resistance of red sea bream (Pagrus major). Fish Physiol Biochem. 2019;45(1):219-230. https://doi.org/10.1007/s10695-018-0556-3

6. Ashouri S, Keyvanshokooh S, Salati A P, Johari S A, Pasha-Zanoosi H. Effects of different levels of dietary selenium nanoparticles on growth performance, muscle composition, blood biochemical profiles and antioxidant status of common carp (Cyprinus carpio). Aquaculture. 2015;446:25-29. https://doi.org/10.1016/j.aquaculture.2015.04.021 
7. Kohshahi A J, Sourinejad I, Sarkheil M, Johari S A. Dietary cosupplementation with curcumin and different selenium sources (nanoparticulate, organic, and inorganic selenium): influence on growth performance, body composition, immune responses, and glutathione peroxidase activity of rainbow trout (Oncorhynchus mykiss). Fish Physiol Biochem. 2019;45(2):793-804. https://doi.org/10.1007/s10695-018-0585-y

8. Liu G X, Jiang G Z, Lu K L, Li X F, Zhou M, Zhang D D, et al. Effects of dietary selenium on the growth, selenium status, antioxidant activities, muscle composition and meat quality of blunt snout bream,Megalobrama amblycephala. Aquaculture Nutrition. 2017;23(4):777-787. https://doi.org/10.1111/anu.12444

9. Xuan X, Li W, Zhu W, Wang S. Effects of different levels of macroalga Gracilaria lemaneiformis on growth performance and feed utilization on the red sea bream, Pagrosomus major. Journal of Applied Phycology. 2019;31(5):3213-3222. https://doi.org/10.1007/s10811-019-01787-9

10. Xuan X, Wen X, Li S, Zhu D, Li Y. Potential use ofmacro-algae Gracilaria lemaneiformis in diets for the black sea bream, Acanthopagrus schlegelii, juvenile. Aquaculture. 2013;412:167-172. https://doi.org/10.1016/j.aquaculture.2013.07.022

11. Luo H, Wang Q, He Z, Wu Y, Long A, Yang Y. Protection of dietary selenium-enriched seaweed Gracilaria lemaneiformis against cadmium toxicity to abalone Haliotis discus hannai. Ecotoxicology and Environmental Safety. 2019;171:398-405. https://doi.org/10.1016/j.ecoenv.2018.12.105

12. Wang L, Xiao J-X, Hua Y, Xiang X-W, Zhou Y-F, Ye L, et al. Effects of dietary selenium polysaccharide on growth performance, oxidative stress and tissue selenium accumulation of juvenile black sea bream, Acanthopagrus schlegelii. Aquaculture. 2019;503:389-395.

https://doi.org/10.1016/j.aquaculture.2019.01.033

13. Zhou N, Long H, Wang C, Yu L, Zhao M, Liu X. Research progress on the biological activities of selenium polysaccharides. Food \& Function. 2020;11(6):4834-4852. https://doi.org/10.1039/c9fo02026h

14. Yu Y-Y, Chen W-D, Liu Y-J, Niu J, Chen M, Tian L-X. Effect of different dietary levels of Gracilaria lemaneiformis dry power on growth performance, hematological parameters and intestinal structure of juvenile Pacific white shrimp (Litopenaeus vannamei). Aquaculture. 2016;450:356-362. https://doi.org/10.1016/j.aquaculture.2015.07.037

15. Yin G, Li W, Lin Q, Lin X, Lin J, Zhu Q, et al. Dietary administration of laminarin improves the growth performance and immune responses in Epinephelus coioides. Fish Shellfish Immunol. 2014;41(2):402-6. https://doi.org/10.1016/j.fsi.2014.09.027

16. Naderi M, Keyvanshokooh S, Salati A P, Ghaedi A. Effects of dietary vitamin E and selenium nanoparticles supplementation on acute stress responses in rainbow trout (Oncorhynchus mykiss) previously subjected to chronic stress. Aquaculture. 2017;473:215-222. https://doi.org/10.1016/j.aquaculture.2017.02.020

17. Lin Y-H, Shiau S-Y. Dietary selenium requirements of juvenile grouper, Epinephelus malabaricus. Aquaculture. 2005;250(1-2):356-363. https://doi.org/10.1016/j.aquaculture.2005.03.022 
18. Zhao L, Qi L, Li C, Li L, Jin L, Yuan J. SVCV impairs mitochondria complex $\nabla$ resulting in accumulation of hydrogen peroxide. Fish \& Shellfish Immunology. 2018;75:58-65. https://doi.org/10.1016/j.fsi.2018.01.053

19. Chen J, Tian M, Guan W, Wen T, Yang F, Chen F, et al. Increasing selenium supplementation to a moderately-reduced energy and protein diet improves antioxidant status and meat quality without affecting growth performance in finishing pigs. J Trace Elem Med Biol. 2019;56:38-45. https://doi.org/10.1016/j.jtemb.2019.07.004

20. Zhan X, Wang M, Zhao R, Li W, Xu Z. Effects of different selenium source on selenium distribution, loin quality and antioxidant status in finishing pigs. Animal Feed Science and Technology. 2007;132(3-4):202-211. https://doi.org/10.1016/j.anifeedsci.2006.03.020

21. Fontagne-Dicharry S, Veron V, Larroquet L, Godin S, Wischhusen P, Aguirre P, et al. Effect of selenium sources in plant-based diets on antioxidant status and oxidative stress-related parameters in rainbow trout juveniles under chronic stress exposure. Aquaculture. 2020;529(0044-8486):735684. https://doi.org/10.1016/j.aquaculture.2020.735684

22. Saffari S, Keyvanshokooh S, Zakeri M, Johari S A, Pasha-Zanoosi H, Mozanzadeh M T. Effects of dietary organic, inorganic, and nanoparticulate selenium sources on growth, hemato-immunological, and serum biochemical parameters of common carp (Cyprinus carpio). Fish Physiology and Biochemistry. 2018;44(4):1087-1097. https://doi.org/10.1007/s10695-018-0496-y

23. Ragaza J A, Koshio S, Mamauag R E, Ishikawa M, Yokoyama S, Villamor S S. Dietary supplemental effects of red seaweed Eucheuma denticulatum on growth performance, carcass composition and blood chemistry of juvenile J apanese flounder, Paralichthys olivaceus. Aquaculture Research. 2015;46(3). https://doi.org/doi:10.1111/are.12211

24. Lambert Y, Dutil J-D. Can simple condition indices be used to monitor and quantify seasonal changes in the energy reserves of cod (Gadus morhua)? NRC Research Press Ottawa, Canada. 1997;54(S1). https://doi.org/doi/10.1139/f96-149

25. Sarkar B, Bhattacharjee S, Daware A, Tribedi P, Krishnani K K, Minhas P S. Selenium Nanoparticles for Stress-Resilient Fish and Livestock. Nanoscale Res Lett. 2015;10(1):371. https://doi.org/10.1186/s11671-015-1073-2

26. Lushchak V I. Environmentally induced oxidative stress in aquatic animals. Aquat Toxicol. 2011;101(1):13-30. https://doi.org/10.1016/j.aquatox.2010.10.006

27. Mechlaoui M, Dominguez D, Robaina L, Geraert P-A, Kaushik S, Saleh R, et al. Effects of different dietary selenium sources on growth performance, liver and muscle composition, antioxidant status, stress response and expression of related genes in gilthead seabream (Sparus aurata). Aquaculture. 2019;507:251-259. https://doi.org/10.1016/j.aquaculture.2019.04.037

28. Passos R, Correia A P, Ferreira I, Pires P, Pires D, Gomes E, et al. Effect on health status and pathogen resistance of gilthead seabream (Sparus aurata) fed with diets supplemented with Gracilaria gracilis. Aquaculture. 2021;531. https://doi.org/10.1016/j.aquaculture.2020.735888 
29. Guo H, Lin W, Wang L, Zhang D, Wu X, Li L, et al. The supplementation of dietary selenium yeast and green tea-derived polyphenols improves antioxidant capacity and immune response in juvenile Wuchang bream under ammonia stress. Aquaculture Research. 2020;51(9):3790-3803. https://doi.org/10.1111/are.14724

30. Harsij M, Kanani H G, Adineh H. Effects of antioxidant supplementation (nano-selenium, vitamin C and $\mathrm{E}$ ) on growth performance, blood biochemistry, immune status and body composition of rainbow trout (Oncorhynchus mykiss) under sub-lethal ammonia exposure. Aquaculture. 2020;521. https://doi.org/10.1016/j.aquaculture.2020.734942

31. Chen H, Li J, Yan L, Cao J, Li D, Huang G Y, et al. Subchronic effects of dietary selenium yeast and selenite on growth performance and the immune and antioxidant systems in Nile tilapia Oreochromis niloticus. Fish Shellfish Immunol. 2020;97:283-293. https://doi.org/10.1016/j.fsi.2019.12.053

32. Guo M, Tang X, Sheng X, Xing J, Zhan W. The effects of IL-1ß, IL-8, G-CSF and TNF-a as molecular adjuvant on the immune response to an $\mathrm{E}$. tarda subunit vaccine in flounder (Paralichthys olivaceus). Fish \& Shellfish Immunology. 2018;77:374-384. https://doi.org/10.1016/j.fsi.2018.04.009

33. Zhao F, Zhuang P, Song C, Shi Z-h, Zhang L-z. Amino acid and fatty acid compositions and nutritional quality of muscle in the pomfret, Pampus punctatissimus. Food Chemistry. 2010;118(2):224-227. https://doi.org/10.1016/j.foodchem.2009.04.110

34. Wang Y, Yu S, Ma G, Chen S, Shi Y, Yang Y. Comparative study of proximate composition and amino acid in farmed and wild Pseudobagrus ussuriensis International Journal of Food Science and Technology. 2014;49(4):983-989. https://doi.org/10.1111/ijfs.12391

\section{Tables}

Table 1 Approximate analysis, additives and actual Se content of different experimental diets. Feeding basic diet (C), supplementing with $0.7 \mathrm{mg} / \mathrm{kg}$ Nano-Se $(\mathrm{N})$ on basic diet, supplementing with $1 \% \mathrm{G}$. lemaneiformis $(\mathrm{G})$, and supplementing with $1 \%$ Nano-Se-enriched $G$. lemaneiformis (NG). 


\begin{tabular}{|lllll|}
\hline Proximate analysis $(\geq \%)$ & Group C & Group N & Group G & Group NG \\
\hline Crude protein & $52.06 \pm 0.00$ & $52.17 \pm 0.12$ & $52.52 \pm 0.30$ & $52.49 \pm 0.21$ \\
\hline Crude lipid & $11.45 \pm 0.21$ & $11.45 \pm 0.35$ & $11.35 \pm 0.64$ & $11.25 \pm 0.35$ \\
\hline Crude fiber & $16.00 \pm 0.00$ & $16.00 \pm 0.00$ & $17.00 \pm 0.00$ & $18.00 \pm 0.00$ \\
\hline Crude ash & $13.55 \pm 0.07$ & $13.70 \pm 0.00$ & $13.65 \pm 0.07$ & $13.80 \pm 0.00$ \\
\hline Moisture & $6.55 \pm 0.07$ & $6.75 \pm 0.07$ & $6.40 \pm 0.00$ & $6.50 \pm 0.00$ \\
\hline Calcium & $1.19 \pm 0.01$ & $1.20 \pm 0.03$ & $1.21 \pm 0.05$ & $1.21 \pm 0.01$ \\
\hline Phosphorus & $1.85 \pm 0.01$ & $1.92 \pm 0.05$ & $1.93 \pm 0.00$ & $1.95 \pm 0.04$ \\
\hline Nano-Se $(\mathrm{mg} \mathrm{Se} / \mathrm{kg})$ & $0.00 \pm 0.00$ & $0.70 \pm 0.00$ & $0.00 \pm 0.00$ & $0.00 \pm 0.00$ \\
\hline G. lemaneiformis $(\mathrm{g} / \mathrm{kg})$ & $0.00 \pm 0.00$ & $0.00 \pm 0.00$ & $10.00 \pm 0.00$ & $0.00 \pm 0.00$ \\
\hline Nano-Se-enriched G. lemaneiformis $(\mathrm{g} / \mathrm{kg})$ & $0.00 \pm 0.00$ & $0.00 \pm 0.00$ & $0.00 \pm 0.00$ & $10.00 \pm 0.00$ \\
\hline Se content $(\mathrm{mg} \mathrm{Se} / \mathrm{kg})$ & $0.14 \pm 0.01$ & $0.82 \pm 0.03$ & $0.16 \pm 0.01$ & $0.82 \pm 0.04$ \\
\hline
\end{tabular}

Table 2 Synthetic oligonucleotides used in this study.

\begin{tabular}{|lll|}
\hline Primer & Sequence $\left(5^{\prime}-3^{\prime}\right)$ & Genbank No. \\
\hline IgM-F & GGAAAAGGACTGGAGTGGATTG & GU988694.1 \\
\hline IgM-R & GCTGTTGTCTGTGGAGATGGTG & \\
\hline IL8-F & AAGTTTGCCTTGACCCCGAA & FJ913064.1 \\
\hline IL8-R & TGAAGCAGATCTCTCCCGGT & \\
\hline TNF-a-F & GGATCTGGCGCTACTCAGAC & FJ009049.1 \\
\hline TNF-a-R & CGCCCAGATAAATGGCGTTG & \\
\hline
\end{tabular}

Table 3 Effect of Nano-Se, seaweed G. lemaneiformis and Nano-Se-enriched G. lemaneiformis on Se content of grouper. $(\mathrm{mg} / \mathrm{kg})$. Feeding basic diet (C), supplementing with $0.7 \mathrm{mg} / \mathrm{kg} \mathrm{Nano-Se}(\mathrm{N})$ on basic diet, supplementing with $1 \%$ G. lemaneiformis (G), and supplementing with $1 \%$ Nano-Se-enriched $G$. lemaneiformis (NG). 


\begin{tabular}{|lllll|}
\hline \multicolumn{2}{|c}{ C } & N & G & NG \\
\hline Muscle Se & $0.02 \pm 0.03^{c}$ & $0.64 \pm 0.02^{\mathrm{b}}$ & $0.03 \pm 0.01^{\mathrm{c}}$ & $0.83 \pm 0.03^{\mathrm{a}}$ \\
\hline Hepatic Se & $0.53 \pm 0.03^{\mathrm{c}}$ & $1.09 \pm 0.02^{\mathrm{b}}$ & $0.54 \pm 0.01^{\mathrm{c}}$ & $1.85 \pm 0.03^{\mathrm{a}}$ \\
\hline
\end{tabular}

Notes: Values are presented as the mean \pm SD. Different superscript letters are obviously different among 4 groups at the same sampling time $(p<0.05)$ based on one-way ANOVA followed by LSD.

Table 4 Effect of Nano-Se, G. lemaneiformis and Nano-Se-enriched G. lemaneiformis on muscle proximate composition of grouper. $(\mathrm{g} / 100 \mathrm{~g})$. Feeding basic diet (C), supplementing with $0.7 \mathrm{mg} / \mathrm{kg}$ Nano$\mathrm{Se}(\mathrm{N})$ on basic diet, supplementing with $1 \% \mathrm{G}$. lemaneiformis $(\mathrm{G})$, and supplementing with $1 \%$ Nano-Seenriched G. lemaneiformis (NG).

\begin{tabular}{|lllll|}
\hline & $\mathrm{C}$ & $\mathrm{N}$ & $\mathrm{G}$ & $\mathrm{NG}$ \\
\hline moisture & $77.00 \pm 0.20^{\mathrm{a}}$ & $76.39 \pm 0.61^{\mathrm{b}}$ & $77.07 \pm 0.50^{\mathrm{a}}$ & $76.37 \pm 0.74^{\mathrm{b}}$ \\
\hline ash & $1.93 \pm 0.23^{\mathrm{a}}$ & $1.73 \pm 0.15^{\mathrm{a}}$ & $1.90 \pm 0.10^{\mathrm{a}}$ & $1.87 \pm 0.06^{\mathrm{a}}$ \\
\hline lipid & $2.00 \pm 0.01^{\mathrm{b}}$ & $2.80 \pm 0.15^{\mathrm{a}}$ & $2.10 \pm 0.10^{\mathrm{b}}$ & $2.80 \pm 0.26^{\mathrm{a}}$ \\
\hline protein & $19.57 \pm 0.9^{\mathrm{b}}$ & $19.20 \pm 0.87^{\mathrm{b}}$ & $18.87 \pm 0.95^{\mathrm{b}}$ & $22.47 \pm 0.15^{\mathrm{a}}$ \\
\hline
\end{tabular}

Notes: Values are presented as the mean \pm SD. Different superscript letters are obviously different among 4 groups at the same sampling time $(p<0.05)$ based on one-way ANOVA followed by LSD.

Table 5 Effect of Nano-Se, G. lemaneiformis and Nano-Se-enriched G. lemaneiformis on amino acids composition of grouper. (\%, wet weight). Feeding basic diet (C), supplementing with $0.7 \mathrm{mg} / \mathrm{kg}$ Nano-Se $(\mathrm{N})$ on basic diet, supplementing with $1 \% \mathrm{G}$. lemaneiformis $(\mathrm{G})$, and supplementing with $1 \%$ Nano-Seenriched G. lemaneiformis (NG). 


\begin{tabular}{|c|c|c|c|c|}
\hline & C & $\mathrm{N}$ & G & NG \\
\hline Histidine His & $0.28 \pm 0.07^{a}$ & $0.29 \pm 0.01^{a}$ & $0.31 \pm 0.01^{a}$ & $0.32 \pm 0.03^{a}$ \\
\hline Isoleucine Ile & $0.76 \pm 0.05^{b}$ & $0.95 \pm 0.01^{\mathrm{a}}$ & $0.74 \pm 0.01^{b}$ & $0.93 \pm 0.01^{\mathrm{a}}$ \\
\hline Valine Val ${ }^{\#}$ & $0.76 \pm 0.03^{d}$ & $1.05 \pm 0.02^{b}$ & $0.86 \pm 0.01^{c}$ & $1.14 \pm 0.01^{\mathrm{a}}$ \\
\hline Aspartic acid Asp* & $1.80 \pm 0.32^{b}$ & $2.27 \pm 0.01^{\mathrm{a}}$ & $1.65 \pm 0.15^{b}$ & $2.33 \pm 0.03^{a}$ \\
\hline Threonine Thr" & $0.66 \pm 0.04^{d}$ & $1.01 \pm 0.01^{\mathrm{b}}$ & $0.79 \pm 0.02^{c}$ & $1.12 \pm 0.01^{\mathrm{a}}$ \\
\hline Serine Ser & $0.79 \pm 0.04^{\mathrm{a}}$ & $0.75 \pm 0.01^{b}$ & $0.62 \pm 0.01^{d}$ & $0.70 \pm 0.01^{c}$ \\
\hline Leucine Leu ${ }^{\#}$ & $1.67 \pm 0.11^{b c}$ & $1.73 \pm 0.01^{\mathrm{ab}}$ & $1.62 \pm 0.05^{c}$ & $1.78 \pm 0.01^{\mathrm{a}}$ \\
\hline Tyrosine Tyr & $0.61 \pm 0.05^{b}$ & $0.68 \pm 0.01^{a}$ & $0.52 \pm 0.02^{c}$ & $0.65 \pm 0.03^{\mathrm{ab}}$ \\
\hline Lysine Lys ${ }^{\#}$ & $1.81 \pm 0.10^{b}$ & $2.01 \pm 0.01^{a}$ & $1.76 \pm 0.04^{b}$ & $2.09 \pm 0.01^{\mathrm{a}}$ \\
\hline Arginine Arg & $1.35 \pm 0.08^{\mathrm{a}}$ & $1.19 \pm 0.06^{b}$ & $1.32 \pm 0.02^{\mathrm{a}}$ & $1.23 \pm 0.01^{\mathrm{b}}$ \\
\hline Glutamic acid Glu* & $2.85 \pm 0.16^{c}$ & $3.25 \pm 0.05^{\mathrm{ab}}$ & $3.15 \pm 0.10^{b}$ & $3.41 \pm 0.01^{\mathrm{a}}$ \\
\hline Glycine Gly* & $1.10 \pm 0.02^{\mathrm{a}}$ & $1.21 \pm 0.02^{b}$ & $1.38 \pm 0.10^{a}$ & $1.31 \pm 0.01^{\mathrm{a}}$ \\
\hline Proline Pro & $0.62 \pm 0.01^{b}$ & $0.91 \pm 0.01^{\mathrm{a}}$ & $0.57 \pm 0.03^{c}$ & $0.64 \pm 0.01^{b}$ \\
\hline Methionine Met ${ }^{\#}$ & $0.57 \pm 0.03^{c}$ & $0.65 \pm 0.01^{b}$ & $0.56 \pm 0.02^{c}$ & $0.75 \pm 0.02^{\mathrm{a}}$ \\
\hline Alanine Ala* & $1.13 \pm 0.04^{c}$ & $1.25 \pm 0.04^{b}$ & $1.06 \pm 0.03^{d}$ & $1.42 \pm 0.03^{\mathrm{a}}$ \\
\hline Phenylalanine Phe ${ }^{\#}$ & $0.80 \pm 0.00^{b}$ & $0.84 \pm 0.04^{\mathrm{ab}}$ & $0.85 \pm 0.01^{\mathrm{a}}$ & $0.83 \pm 0.03^{\mathrm{ab}}$ \\
\hline $\mathrm{W}_{\text {DAA }}$ & $6.88 \pm 0.43^{c}$ & $7.99 \pm 0.04^{b}$ & $7.24 \pm 0.14^{c}$ & $8.48 \pm 0.07^{a}$ \\
\hline $\mathrm{W}_{\text {TAA }}$ & $16.95 \pm 0.34^{d}$ & $24.58 \pm 0.52^{a}$ & $17.64 \pm 0.49^{c}$ & $23.10 \pm 2.16$ \\
\hline $\mathrm{W}_{\text {EAA }}$ & $7.02 \pm 0.33^{c}$ & $8.23 \pm 0.06^{b}$ & $7.17 \pm 0.10^{c}$ & $8.63 \pm 0.03^{a}$ \\
\hline $\mathrm{W}_{\mathrm{NEAA}}$ & $9.93 \pm 0.20^{d}$ & $11.79 \pm 0.09^{b}$ & $10.59 \pm 0.19^{c}$ & $12.01 \pm 0.10^{\mathrm{a}}$ \\
\hline $\mathrm{W}_{\mathrm{DAA}} / \mathrm{W}_{\mathrm{TAA}}$ & $0.41 \pm 0.02^{\mathrm{a}}$ & $0.40 \pm 0.00^{a}$ & $0.41 \pm 0.00^{a}$ & $0.41 \pm 0.00^{a}$ \\
\hline $\mathrm{W}_{\text {EAA }} / \mathrm{W}_{\mathrm{TAA}}$ & $0.41 \pm 0.01^{\mathrm{ab}}$ & $0.41 \pm 0.00^{\mathrm{ab}}$ & $0.40 \pm 0.00^{b}$ & $0.42 \pm 0.00^{\mathrm{a}}$ \\
\hline $\mathrm{W}_{\text {EAA }} / \mathrm{W}_{\mathrm{NEAA}}$ & $0.71 \pm 0.04^{\mathrm{ab}}$ & $0.70 \pm 0.00^{\mathrm{ab}}$ & $0.68 \pm 0.01^{b}$ & $0.72 \pm 0.01^{a}$ \\
\hline
\end{tabular}

Notes: Tryptophan is destroyed in the acid hydrolysis, so it is not detected. 
Values are presented as the mean $\pm S D$. Different superscript letters are obviously different among 4 groups at the same sampling time $(p<0.05)$ based on one-way ANOVA followed by LSD. WTAA is total amino acids (TAA); $W_{\text {EAA }}$ is total essential amino acids (EAA); $W_{\text {NEAA }}$ is non-essential amino acids (NEAA); $W_{\text {DAA }}$ is total delicious amino acids (DAA). *means delicious amino acids, "means essential amino acids.

\section{Figures}

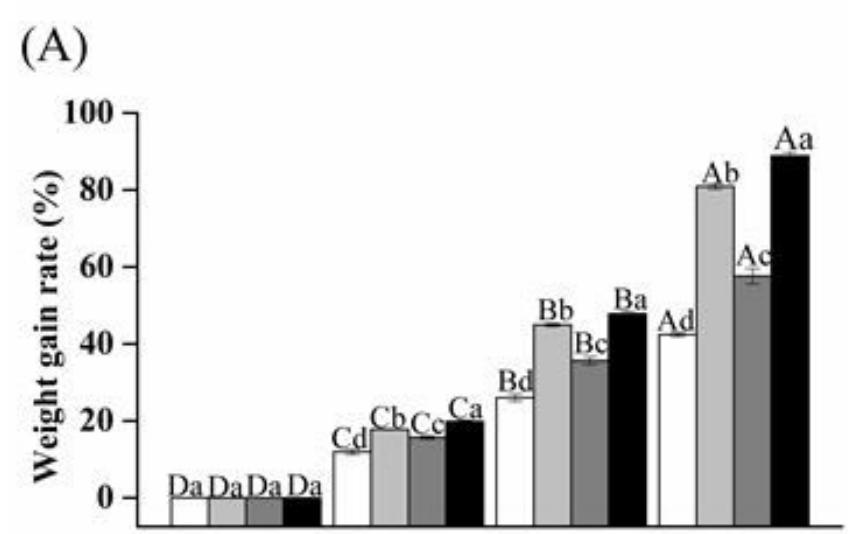

(B)

(C)
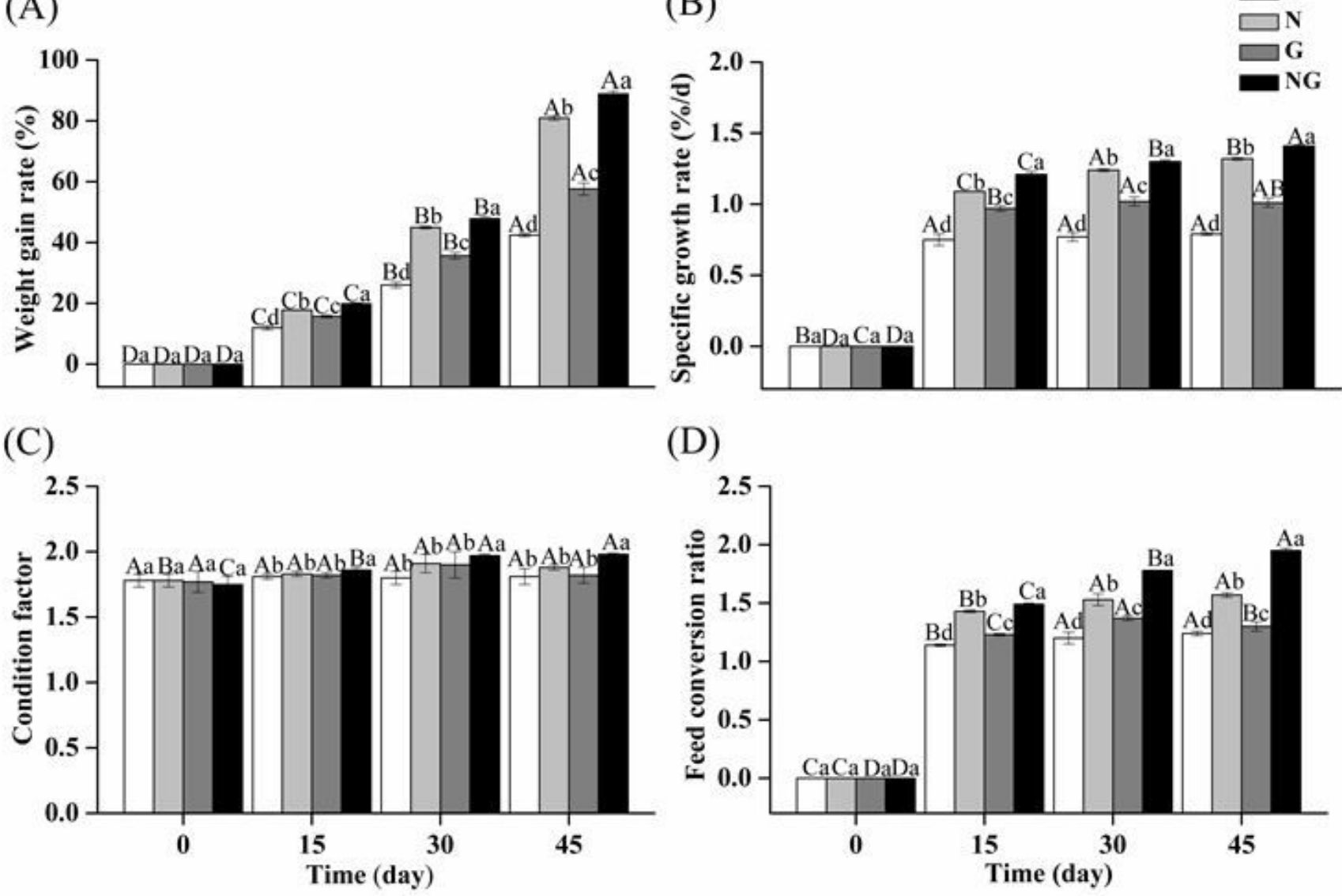

(D)

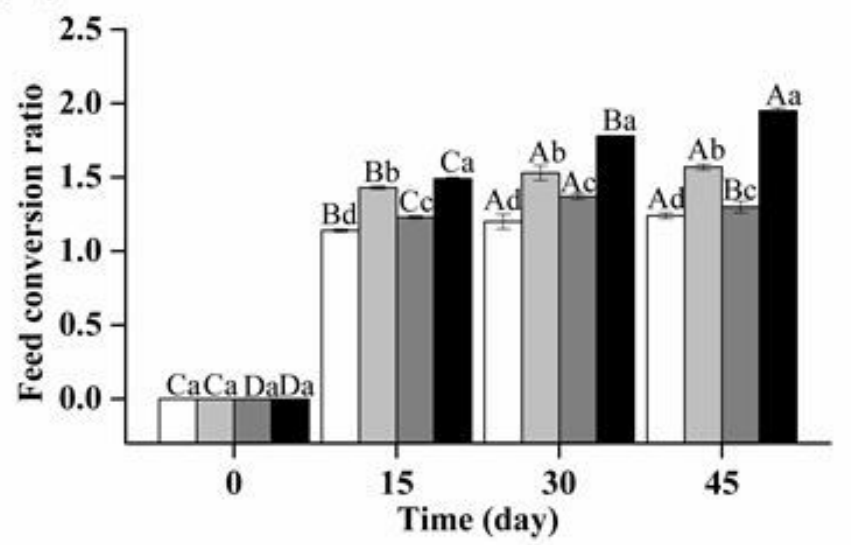

Figure 1

Effects of Nano-Se, G. lemaneiformis and Nano-Se-enriched G. lemaneiformis on the WGR (A), SGR (B), CF (C) and FCR (D) of grouper on the 0th, 15th, 30th and 45th sampling day. Various uppercase letters indicate remarkable differences among test time points in the same group, different lowercase letters are represent significant difference among four groups at the same sampling time $(p<0.05)$ based on oneway ANOVA followed by LSD. The four groups were feeding basic diet (C), supplementation with 0.7 
$\mathrm{mg} / \mathrm{kg}$ Nano-Se $(\mathrm{N})$ on basic diet, supplementation with $1 \% \mathrm{G}$. lemaneiformis $(\mathrm{G})$, and supplementation with $1 \%$ Nano-Se-enriched G. lemaneiformis (NG), respectively.

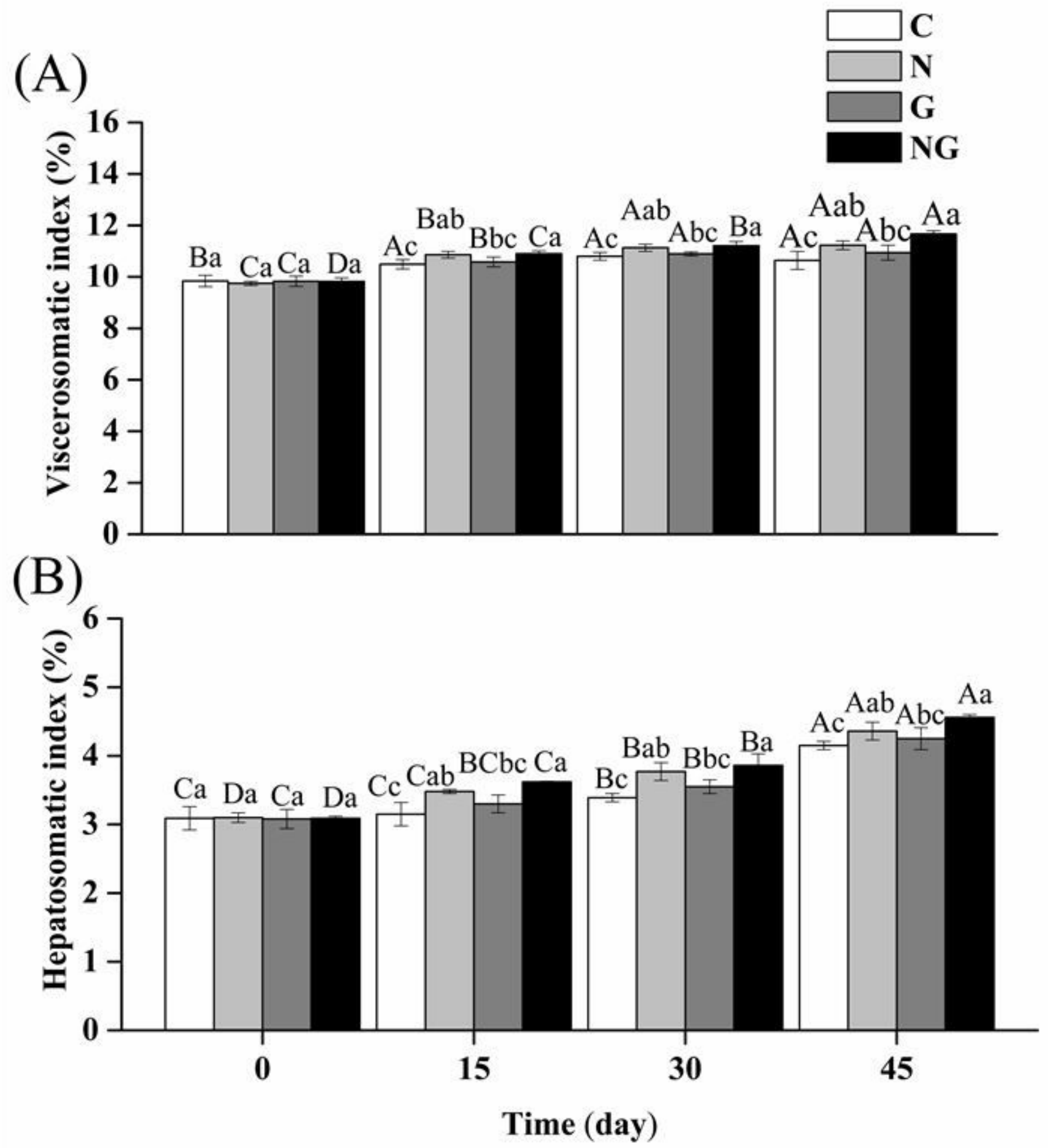

Figure 2

Effects of Nano-Se, G. lemaneiformis and Nano-Se-enriched G. lemaneiformis on the VSI (A) and HSI (B) of grouper on the 0th, 15th, 30th and 45th sampling day. Various uppercase letters indicate remarkable differences among test time points in the same group, different lowercase letters represent significant 
difference among four groups at the same sampling time $(p<0.05)$ based on one-way ANOVA followed by LSD. The four groups were feeding basic diet (C), supplementation with $0.7 \mathrm{mg} / \mathrm{kg} \mathrm{Nano-Se}(\mathrm{N})$ on basic diet, supplementation with $1 \%$ G. lemaneiformis (G), and supplementation with $1 \%$ Nano-Seenriched $\mathrm{G}$. lemaneiformis (NG), respectively.
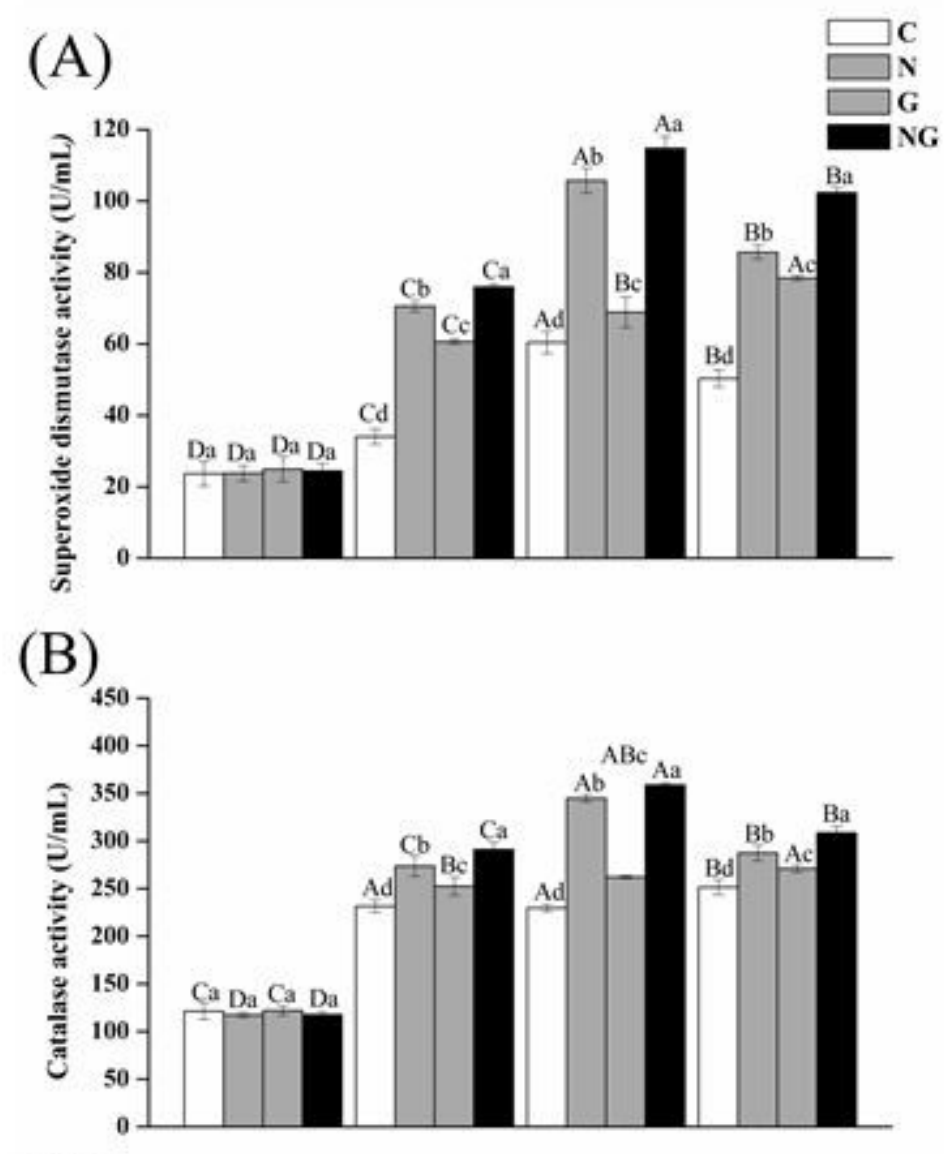

(C)

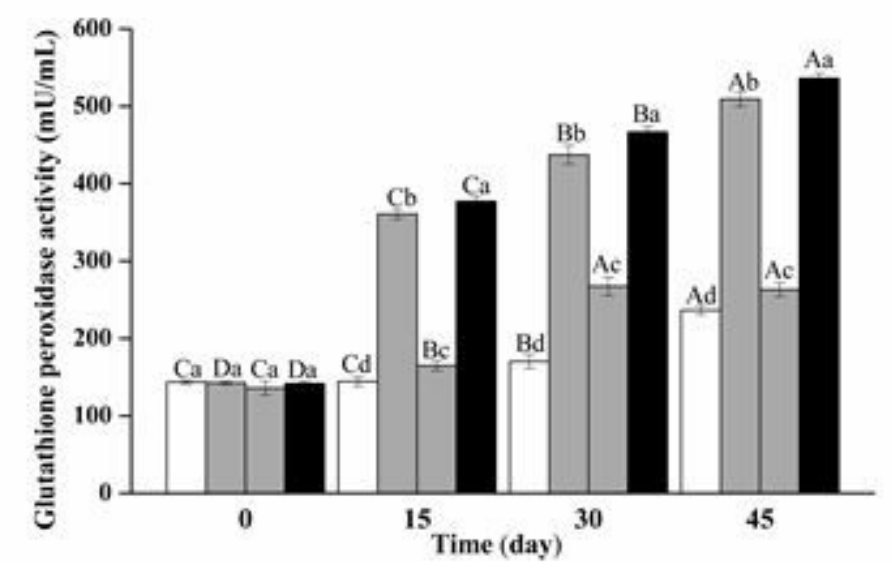

\section{Figure 3}

Effects of Nano-Se, G. lemaneiformis and Nano-Se-enriched G. lemaneiformis on the SOD (A), CAT (B) and GPx (C) of grouper on the 0th, 15th, 30th and 45th sampling day. Various uppercase letters indicate 
remarkable differences among test time points in the same group, different lowercase letters are represent significant difference among four groups at the same sampling time $(p<0.05)$ based on oneway ANOVA followed by LSD. The four groups were feeding basic diet (C), supplementation with 0.7 $\mathrm{mg} / \mathrm{kg}$ Nano-Se $(\mathrm{N})$ on basic diet, supplementation with $1 \% \mathrm{G}$. lemaneiformis (G), and supplementation with $1 \%$ Nano-Se-enriched G. lemaneiformis (NG), respectively.
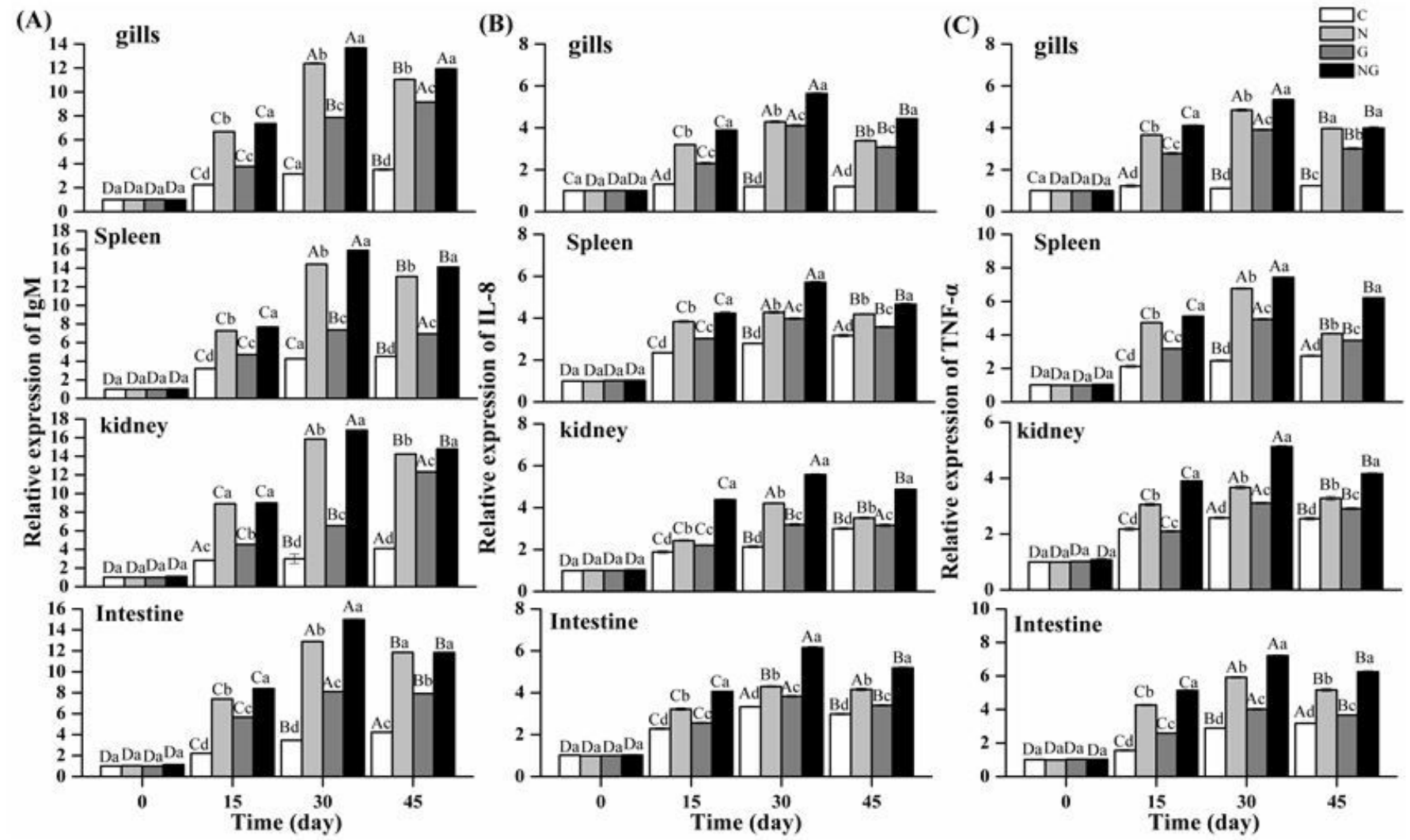

Figure 4

Effects of Nano-Se, G. lemaneiformis and Nano-Se-enriched G. lemaneiformis on the IgM (A), IL-8 (B) and TNF-a(C) of grouper on the 0th, 15th, 30th and 45th sampling day. Various uppercase letters indicate remarkable differences among test time points in the same group, different lowercase letters are represent significant difference among four groups at the same sampling time $(p<0.05)$ based on oneway ANOVA followed by LSD. The four groups were feeding basic diet (C), supplementation with 0.7 $\mathrm{mg} / \mathrm{kg}$ Nano-Se $(\mathrm{N})$ on basic diet, supplementation with $1 \% \mathrm{G}$. lemaneiformis (G), and supplementation with $1 \%$ Nano-Se-enriched G. lemaneiformis (NG), respectively.

\section{Supplementary Files}

This is a list of supplementary files associated with this preprint. Click to download. 
- Supplementarymaterials.doc

Page 22/22 